\title{
Augmented Reality Musical App to Support Children's Musical Education
}

\author{
Bruno Lemos $^{1}$, Ana G. D. Corrêa ${ }^{1,2, *}$, Marilena Nascimento ${ }^{3}$, Roseli D. Lopes ${ }^{1}$ \\ ${ }^{1}$ Department of Electrical Engineering, University of São Paulo, Brazil \\ ${ }^{2}$ Faculty of Computer Science and Information Technology, Mackenzie Presbyterian University, Brazil \\ ${ }^{3}$ Espaço Terapêutico de Medicina Integrada - COLMEIA, Brazil
}

Copyright $\odot 2017$ by authors, all rights reserved. Authors agree that this article remains permanently open access under the terms of the Creative Commons Attribution License 4.0 International License

\begin{abstract}
This article presents an Augmented Reality App to support musical learning of children. The App works by verifying whether sequences of musical notes of the melody are correctly colored in a printed pentagram (target). Meanwhile, an animation in Augmented Reality with a 3D character dancing to the rhythm of the melody is initiated. The App has been previously tested with six children and the results point towards the great potential of this tool to improve the process of children's musical education.
\end{abstract}

Keywords Augmented Reality, Mobile Technologies, Musical Education

\section{Introduction}

The use of mobile application by children has become a fertile field of investigation. Recognized as digital native for their innate abilities with the technologies, these children are becoming more and more consumers and producers of technological culture, especially with mobile devices such as smartphones and tablets [1, 2].

Augmented Reality (AR) technology allows incorporate visual and auditory information into the real world in real time. It is truly starting to support a number of interactive tools, especially for navigation aids. There are already RA-based learning applications supported by webcams and portable and desktop computers that act as a gateway between the real world and the virtually enriched world. Mobile RA is at an early stage and takes its first steps in the educational process, but it has a huge potential still unexplored. This article is focused on the potential of AR apps to support the process of the children's musical education.

Think about music education in childhood is important, because children have great potential to develop musically, which should be encouraged from an early age. According to
[3], musical practice at this stage is essential to the child's development process as it helps in concentration, motor coordination, socialization, auditory acuity, emotional balance, among others. As children participate in musical activities their interest in the registration forms grows, that is, the writing referred to as musical notation.

One of the strategies used in teaching musical notation for children is to color the musical notes pentagram. This allows kids to learn these notes and also to know their right position in the pentagram. Based on this, they begin to read and to write songs of the infant universe. An example can be found in the market, at newsstands, in magazines with sheet music to paint or children's music books with keyboards attached, found in toy stores such as "Disney Songs for Classical Piano" [4].

Musicalization therefore, is a way to teach music to children by interactive and facilitating activities that can be pleasant. In order to support children's musicalization by coloring the pentagram notes, an AR Musical app was developed. The application is able to check whether sequences of musical notes were correctly colored in a printed pentagram on paper. If the sequence of musical notes is correct, the application shows an animation of a 3D character dancing to the music on the pentagram. The application can be used on Android phones and tablets. The idea is to stimulate children's interest by musical activities in the digital world. The application was tested with six children. The results indicate the potential use of this tool for children's musical education.

The article is organized as follows: the next section introduces the use of mobile applications in education, especially in music education; Characterizes augmented reality systems and discusses their use in education, presenting some examples of mobile augmented reality applications; Section III shows the development of the Music App; Next, section IV presents the results of the pilot test applied in a real musical education context; Finally, section $\mathrm{V}$ presents some final considerations of this work and future 
perspectives.

\section{Background}

\subsection{Mobile Learning}

The term "Mobile Learning" or M-Learning is designed to represent a type of distance learning (unassisted) that has had a great impact on how people learn, causing a new educational paradigm. Access to mobile technologies such as tablets, smartphones and e-books connects users to the world instantly, increases access to information, and enables interactivity. And this, especially with children, drastically transforms the way they view the learning process by their own stimulated creativity $[1,2]$.

While the methods of traditional education demand excessive resources: time of a teacher, physical displacement of the involved ones, printed materials, provision of a space for the classes; M-Learning aims to be an increasingly democratized means of access to knowledge. This is because the devices use open platforms, which allows the insertion of applications with educational bias at low cost and with great potential to scale [5].

Unfortunately, before the popularization of mobile devices, they were seen as the great villains of classroom learning. Many teachers have fought against their use by stating that the devices cause distraction and decreased productivity. But at the same time, technology-enabled learning and teaching experts have shed more light on the subject by suggesting that these same distractors can be used as elements of engagement. Traxler [6] explains how this can occur: "While traditional learning happens in exchange for everyday life, M-Learning is part of the user's real life, allowing interaction with information and other users in their day-to-day lives".

The trend towards greater participation of these technologies in people's lives is growing. The phenomenon of the Internet of Things [7] has contributed to make them ubiquitous. It is information that can be accessed anywhere, anytime, connecting the whole world in an exchange of knowledge and collaboration.

Currently, the best example of this universalization of knowledge is the movement of MOOCs (Massive Open Online Courses), such as Coursera [8]. They bring together the most renowned universities in the world and distribute their courses free of charge to anyone who has a device with connectivity and Internet access. They were born under the concept of "Connectivism" and have as their pillars: diverse knowledge; students free to choose when and what to learn; interaction between students and the incentive to solve group problems [9].

This concept can easily be expanded to the infantile world. It is a fact that, every generation, children become more adept of new technologies. According to a survey conducted by CETIC (2015), in Brazil, one in three kids with access to the internet do so by mobile devices. However, we see that this potential is underutilized by comparing this with the data on where and for what these children access the network. The research continues: only $36 \%$ of connected Brazilian children, aged 9 to 10 years, do so at school; While $78 \%$ are on social networks and show great concern about their status in these virtual environments.

Thus, is also emerging the necessity for educators and content creators to engage more with mobile technologies in order to convert children's attention to social networks into productive learning work.

\subsection{Augmented Reality and Mobile Learning}

Coined in 1992 by Caudell [10], the term Augmented Reality referred to the superposition of material presented by a computer to the real world. Even today, the definition remains, in distinction to the term from which it originated: virtual reality.

Augmented Reality (AR) is a technology that enables mixing virtual objects with real scenes, generating a mixed environment, which can be viewed in real time in a display [11]. Devices such as smartphones and tablets are capable of supporting an AR system because they have displays, cameras and a high processing capacity. The operation is simple [11]: the real environment is captured by the camera; then the scene of the real environment and 3D objects are mixed by the AR application and displayed on the device itself.

It is clear then that the emergence of Augmented Reality from Virtual Reality was only possible thanks to technological advances in the areas of image capture, recognition of texture patterns and 3D rendering. The distinction between these two technologies is clear: virtual reality transports the user to the virtual world, thus, it has a predominance of virtual elements; and its augmented version incorporates and superimposes virtual elements on the real world, possessing real-world dominance in the user's view $[11,12]$.

The first devices developed for virtual reality application were the so-called Head Mounted Devices (HMD), in 1966, by Ivan Sutherland, an American computer scientist and Internet pioneer. This device basically obtains an enhanced view of the real environment by superimposing 3D computer generated objects into the real world view (Rolland; Fuchs, 2000). About 50 years later, the most widely used technology for augmented reality applications are mobile devices, such as tablets, laptops, smartphones and other handheld devices [13].

The great advancement of handheld devices compared to HDMs is the presence of these tools in people's daily lives. Tablets and smartphones have functions other than augmented reality which are very useful in everyday life. That way, people in general can enjoy the applications of AR without needing a device exclusively for that. This ease of access has caused a change of mentality, as Billinghurst says, because unlike HDMs, mobile devices "are typically used only for short periods of intensive activity" [14].

For being present in a ubiquitous technology, Augmented Reality certainly wouldn't be left out of the many tools that 
education uses for improving people's learning rate. AR applications have been helping as a motivating tool for children's learning. The newspaper Tokyo Shimbun launched the Tokyo AR, an AR app that aims to encourage children to read [1]. When you point the phone camera to the newspaper marks, the text "emerges" in the screen and animations appear to guide the reading, along with comments and animated title balloons. Similarly, Disney Research has developed an AR app that works along with coloring books. As the colors are added to the paper, the app transforms the texture that was just colored in an AR 3D model. By simply pointing a device to the drawing, the character emerges in three-dimensional form. There are other coloring applications with AR, such as ColorMix, available for Android, and ARKids, an iOS app.

\subsection{Mobile Augmented Reality in Musical Education}

Very little in the literature of Augmented Reality can be found being used to aid in the education and teaching of music. This opens our eyes to the great potential for commercial expansion in this area.

An interesting example is the app developed at Bowling Green State University (USA) [15]. "Prelude", as it was called, is an iOS quiz application for students to learn how to recognize specific music notes and symbols. The iOS game application has two game modes, a "quiz mode" and a "free play mode." Selecting the "quiz mode" option allows the student to take a graded or ungraded quiz created by the teacher. By selecting the "free play" option, the student has free reign to scan any music symbols or notes known by the system (and there are many), and the application will tell them which note or symbol they scanned with their device camera. The application does not require that they take a picture of the scene because it recognizes images in real time from the camera's video feed.

This is a clear example that demonstrates the autonomy that students gain because they do not need a teacher to validate their work. Also, by using the "free play" mode you can learn music at any time of your day, which would have been impractical.

\section{Materials and Methods}

\subsection{Development of AR Musical App}

AR Musical App is an Android application that seeks to make children's music learning more interactive and fun. In short, it checks the ability of children to relate a musical note to their position on the pentagram of a staff and gives visual and auditory feedback to ensure learning.

For the development of the application, software that allows easy integration between the desired functionalities and elements of augmented reality was used. Thus, we chose the Vuforia framework, developed by Qualcomm, which uses Computational View (a field that studies methods to acquire, process and analyze real images and translate to machine language) to recognize and locate flat images or 3D objects and then position objects of augmented reality. However, Vuforia is just an extension that should be added to a game development environment and it is only supported by Unity, which was then chosen for the creation of Musical AR. Unity is a game engine available in Pro (paid) version and free, for personal use, with some limited resources.

A game engine is a set of libraries that bring together tools common to most games, such as graphics engine to render 2D and 3D graphics, physics engine to simulate game physics, and more. In this way, the developer focuses on the specifics of his project.

Still, fundamental elements for applications with Augmented Reality are the 3D models. In the project that this article refers to, FBX extension models (Filmbox) are used to animate the character of the melody, a frog. Finally, because the application's purpose is to aid in musical learning, some WAV extension audio was used to play each musical note, besides the sound of error.

\subsection{How the AR Musical App Works}

First, a blank musical score is given to a child and the challenge is for him to paint each note with the right matching color.

The AR Musical app allows checking whether sequences of notes were correctly colored in a printed pentagram. The pentagram is a graphical system that uses written symbols on a staff of five parallel and equidistant lines and form four spaces between them. Each note is represented in polka dot format; depending on where it is graphed on the staff, it identifies a different sound. The polka dots, or musical notes, must be colored according to a legend of colors that can be defined either by the app or by the user. Thereunto, prior to the use of the app, there must be a previous guidance to instruct the child about musical notation and the app may serve as a workout following this guidance.

After all the notes on the staff are painted, it becomes a target that is recognized by the AR app. When the app is started, the device rear camera is triggered immediately and edges appear on the screen (Figure 1).

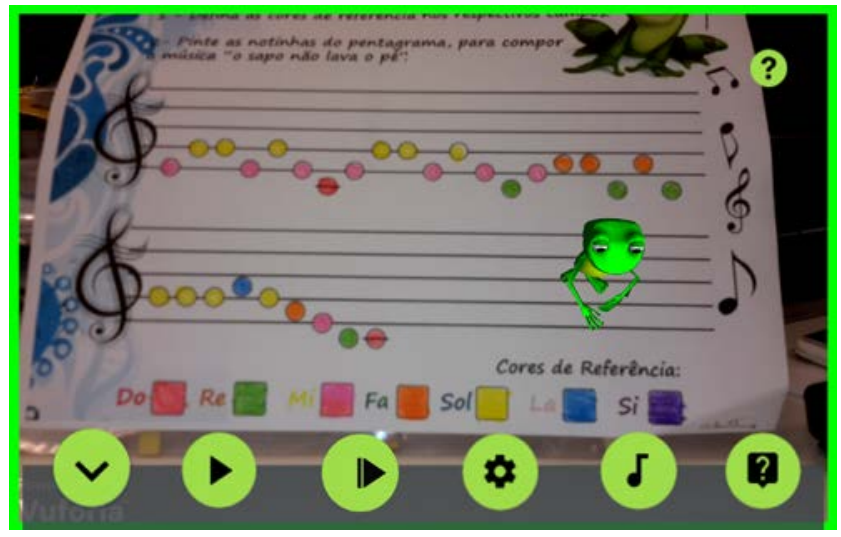

Figure 1. App’s first screen

The app core is based on the identification of: a) a target 
(marker) that drives the 3D model; b) the camera flash for greater accuracy of the color obtained; c) a process of camera auto-focus on the target. The algorithm checks whether the distance, measured in three axes $(\mathrm{x}, \mathrm{y}, \mathrm{z})$, of the camera to the target is within the specifications pre-defined. In case it is, the edges will become green, indicating that the process can be started pressing the "play" button, otherwise the edges turn red (the same happens if the target is lost - Figure 2).

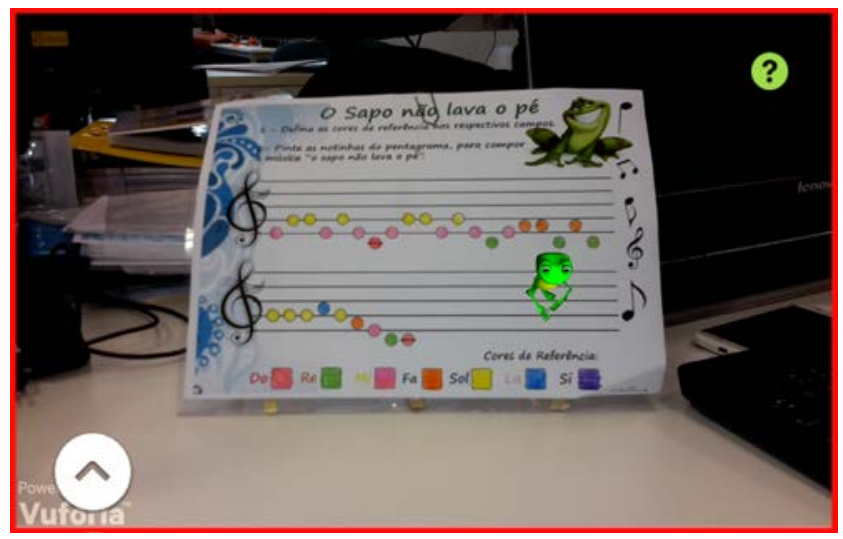

Figure 2. Device too far from the target

By enabling the "play" button, an animation with a character dancing to the music starts while the musical notes on the staff are played. Parallel to that, a sphere will jump over each note in the pentagram to the rhythm of the song illustrating when the notes are right or wrong. If they are properly colored, the sphere becomes green, otherwise it becomes red and an error sound is played. When the character animation is over, the sphere disappears and a new cycle can be started.

Vuforia framework was used for app design. This software allows developing applications in AR integrated with Unity 3D game engine. 3D models in FBX extension (Filmbox) were used in the animations. The audios for playback of musical notes and other sound effects are in WAV format. Lastly, Unity 3D was also used to put together all these files with the algorithm that gives the sequence of colors in the musical score of the pentagram.

Recognizing the target, the position of each musical note on the staff is known by its position relative to the edges of the target. Then, the pixels are scanned and their RGB color is extracted from an area of $10 \times 10$ pixels for each note and stored in a matrix. An average of these 100 pixels is made and the resulting color (RGB) is converted into HSV scale (HUE, Saturation, Value), where HUE indicates from 0 to 360 what color tone was obtained (Figure 3 ).

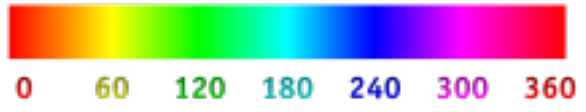

Figure 3. HUE scale
First, the program checks if the color is not white (which should be considered invalid), then the HUE value of the note is compared with the HUE of its specific reference in the legend, which also suffered the same color extraction process. For example, the first note of this project music sheet, Mi, is compared with the third rectangle legend on the target, identified by $\mathrm{Mi}$ and so on. The freedom to paint the legend and the implementation of color extraction are important, because this guarantees that the child can use any material at home to color the musical notes from crayons up to pens. At the end of the color matching process, the $3 \mathrm{D}$ character animation starts and the sphere appears on each note while the sound of the note is played.

If the colors compared are correct, the ball will turn green and the note's melody (for example, C) will be played, otherwise the ball will turn red and an error sound will be played (Figures 4 and 5). At the end, the frog's movement closes and the sphere disappears, so that the process can be started again, with the capture of a new image.

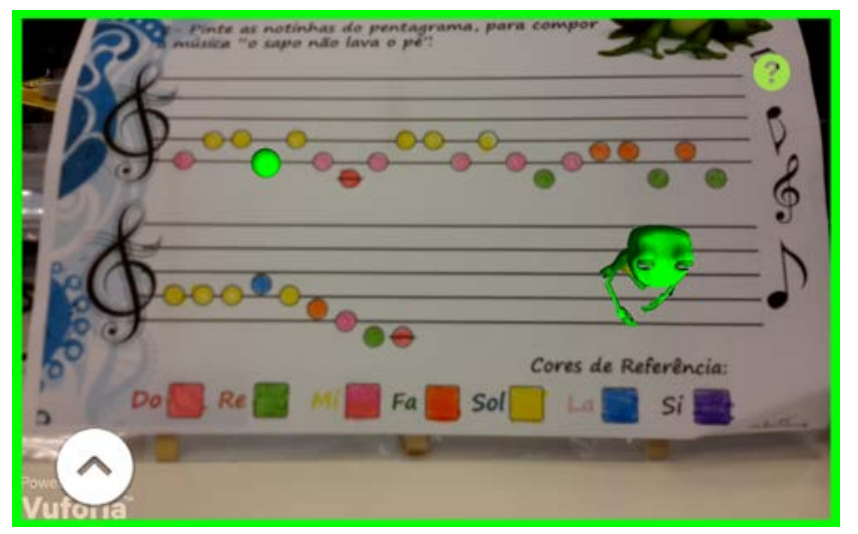

Figure 4. Success case, note painted correctly.

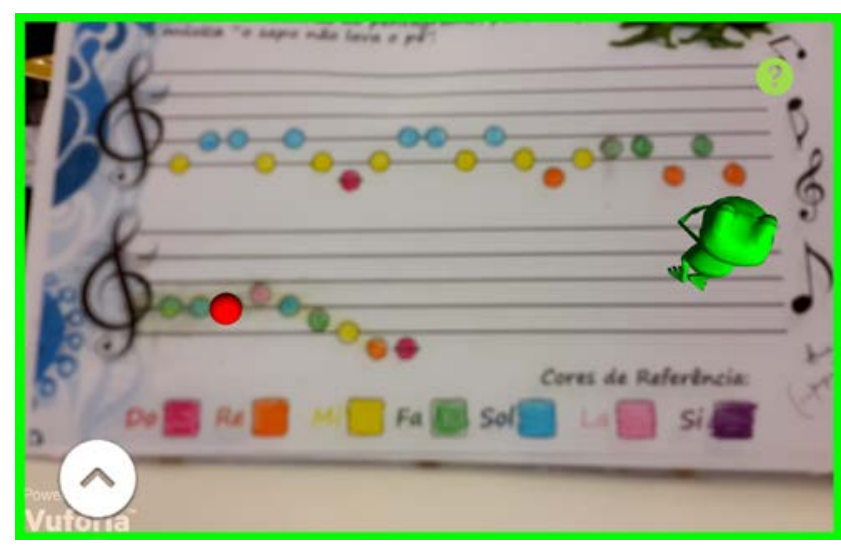

Figure 5. Fail case, note was painted with the wrong color.

There are basically two game modes that can be accessed through the "settings" menu (Figure 6), represented by a button with a gear. 


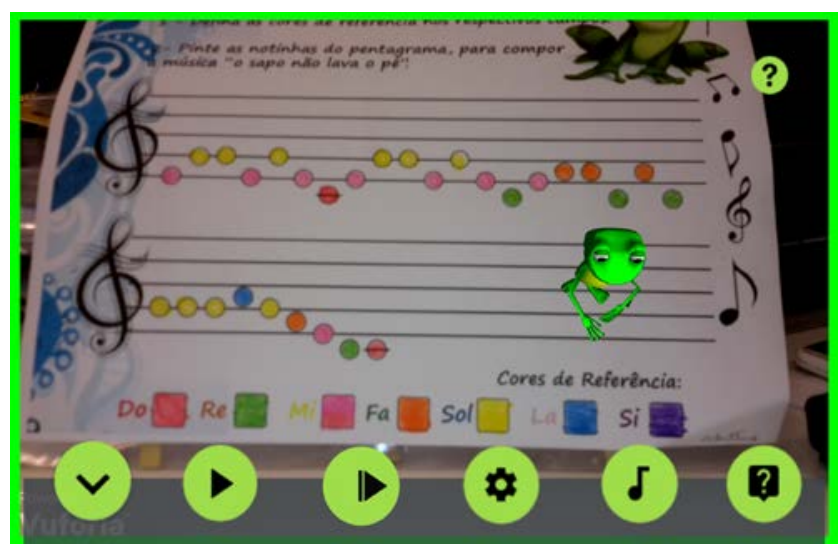

Figure 6. The gear icon represents the Configuration menu.

In standard mode (Figure 7), the subtitle colors are already filled automatically by flattened cubes in AR and it is up to the user to paint each note of the score of the same color as the predefined one in each subtitle rectangle. The free mode follows what was explained in the previous paragraph, giving freedom to choose the reference colors of the legend.

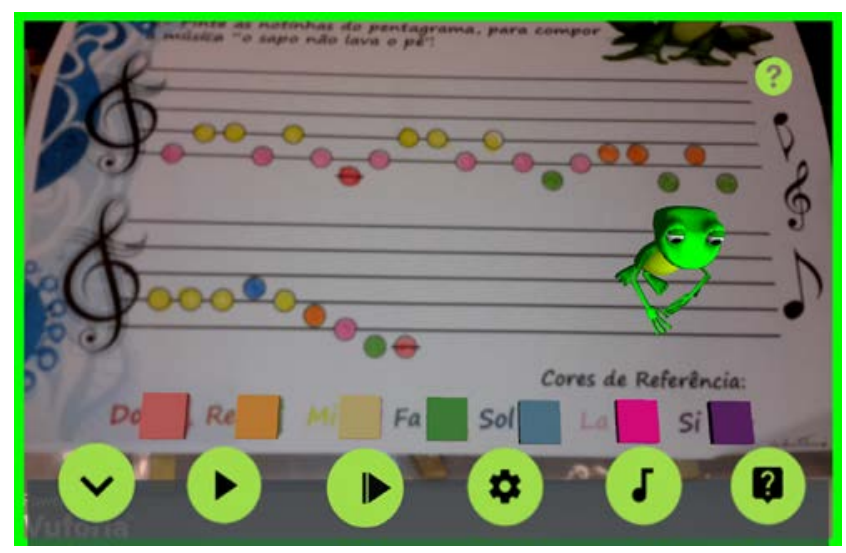

Figure 7. Standard mode, legend have preconfigured colors.

In view of the different user profiles with their respective levels of learning, there is the possibility to increase the speed of the music in up to 2 levels beyond the normal and this process can be done while the music plays or before it starts.

Still in the menu, there are two pop-ups, the "Help" menu that works as a manual, in text, giving instructions for using the application; Also the "Musical Notation" menu, which visually shows the correct position of each note (from $\mathrm{C}$ to $\mathrm{S}$ ) in the score. As a last aid tool to improve usability, there is a button with a question mark (“?”) in the top right corner of the screen, pressing it brings you to a tutorial on 9 navigable images (back and forward) to explain the functionality of each button on the main menu.

\subsection{Tech Specifications}

As mentioned in previous topics, the application was developed using Unity3D framework and Vuforia
Framework, with the following specifications:

- Platforms: Android;

- $\quad$ Operation System Versions: Android 4.1+ (SDK 17);

- $\quad$ Development framework: Unity3D 5.51f1;

- $\quad$ Plugins and add-ons: Vuforia 6.2 SDK for Unity;

- $\quad$ Scripts Code language: C\#;

- Services, servers and databases: There are no connection with external servers in the current version; database is limited to local storage;

- $\quad$ Maintenance needs: Add support to iOS; enhance app performance and lower power consumption;

- Availability: App is currently available only through apk install (not available on Play Store);

The Unity3D architecture implementation works as represented bellow:

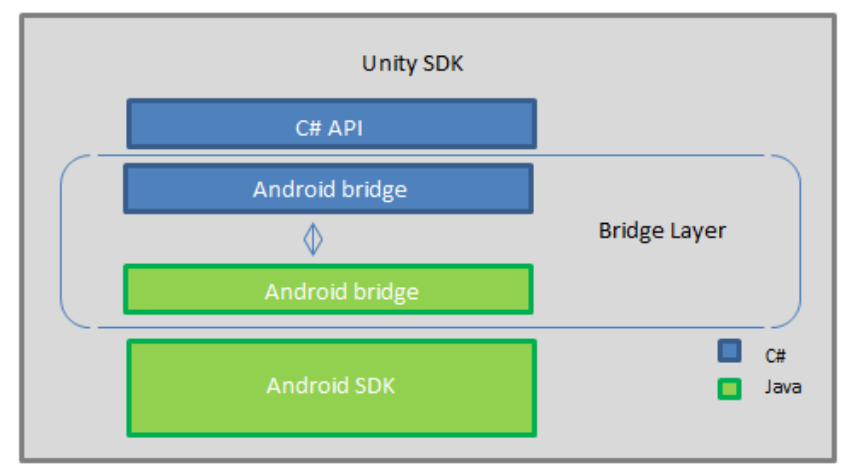

Figure 8. Unity Architecture

\subsection{Learning Process}

In everyday life, one of the strategies to remember learning is to repeat it several times. For information to be stored in memory it is necessary to "create a history" or mental image of what one wants to remember or learn. In this sense, the relationship between color and sounds leads to learning facilitating or reinforcing the retention of information [16].

The AR Musical app has as learning strategy to color the musical note, associating the sounds with colors. The matching of the sound emitted goes against assertiveness, initially from a small melody or a musical phrase of public domain and, later, increasing its complexity.

The student may recognize whether he has hit or miss the melody with his ears. If the student identifies an error, then he can through the colors make sure and find the error in the musical staff, thus promoting his learning. The auditory and the visual canal reinforce the learning, circularly (feedback), leaning on one another in order to identify the musical note (sound) in its proper position in the pentagram (colors).

For the evolution of musical learning it is necessary to offer new musical phrases known or created by the learner where step by step will be inserted new challenges with other elements of musical pedagogy such as: pauses, musical figures with different time values among other concepts 
proper to musical pedagogy.

\subsection{A preliminary Study of Viability}

A pilot test was conducted with the target audience to validate the viability of the application in children's music lessons. Participants were selected by an expert in music education by invitation to parents of friends and acquaintances. A free and informed consent form was signed by the parents or guardians of each participant included in the research, assuring them of the anonymity of the information.

Pretest and posttest evaluation questionnaires were developed to assist in data collection. The pretest questionnaire was used to collect demographic data (age, gender and schooling) and information about the experience with music, mobile devices and augmented reality. The posttest questionnaire was applied after the application was used to collect data on the satisfaction of the interaction with the product. In addition, an observation questionnaire was completed by the specialist in order to get their impressions about the experience, as well as criticisms and suggestions for improvements. At the end of the session the participants won a participation toast. The tests were performed individually and on alternate days upon parental availability. All participants used the same tablet model: Samsung Galaxy Tab 4 10.1 SM-T530 Android 4.4 16GB.

\section{Results and Discussion}

Six children, mean age 6 years, 3 boys and 3 girls participated in this study. All participants reported using tablets and smartphones from parents and/or family members to play with moderate frequency ( 2 to 3 times a week), but none of them had ever interacted with augmented reality technology. As for musical knowledge, none of the children had had contact with music education classes.

As none of the participants had previous contact with augmented reality, which was expected, given the recent introduction of this technology in education and entertainment, it was necessary to present the concept through a demonstration in the study tablet itself. The selected application was ARKids [1] which shows a 3D character of a cat from a colored target. The participants had time to manipulate the device in order to "learn" to visualize the 3D object in different perspectives in the real environment. Children who underwent the experience of using $\mathrm{AR}$, by the time they engaged with the application, they required little or no interference from the applicator. Preliminary instructions were enough for the children to interact with the application. This fact leads us to believe that through their inner experiences, they were able to promote their own insights, and whenever positive facts of learning occurred, they expressed happiness contentment by being able to "guess" the challenge posed by app.
Before using the application it was also necessary to introduce the concepts of musical writing from the representation of the musical notes in the musical score. We leave the expert free to decide the best strategy to introduce these concepts needed to use the application. First, the specialist presented the musical notes and their respective positions in the pentagram. He then handed out a blank sheet with a pentagram so the participant could represent the musical notes on it. It was a type of training.

After the assimilation of the necessary musical concepts, the test with the AR Musical application started. Each participant received a blank target with the song "O Sapo não lava o pé" (in literal translation "The Toad does not wash his foot"). The participant was asked to set the colors in the caption and then to paint the notes of the musical staff according to the legend defined by him. Then the participant received the tablet with AR Musical app and was instructed to frame the target in the field of view of the camera, as they had done with the ARKids. Finally, the animation of a dancing frog began in augmented reality.

The excitement of the participants showed that they were very motivated by the activity they had just completed and that they effectively learned the positions of the musical notes on the staff. One of the children showed self-criticism: because of the sonic result, she realized that she had made a mistake. Spontaneously, then, she searched for a bug in the pentagram, asked for another coloring sheet, did the autocorrect without the help guide of the app. The song was played again and correctly.

The positive point for using AR Musical is that from internal experiences it was possible to learn established between the user and the application. Many times those who are willing to teach have their own terminology and the digital tool has been showing us a language for learning that has direct access to the user, especially for the younger ones, eg babies use of tablets. Another positive point is that by bringing learning experiences you can also stimulate: visual, auditory and motor skills. In addition, internal content pre-experienced by memory, reinforces the new learning that are facilitative strategies, since they stimulate several channels of learning at the same time.

\section{Conclusions and Future Works}

Learning the notes on the staff using the AR Musical app is a facilitator since it is a general consensus, among music teachers, that one of the major barriers to be transposed into formal music is learning the notes on the staff.

New technologies that use augmented reality can help reduce the initial difficulties involved in learning music by reducing these barriers of traditional teaching materials. Also, it is a cheap and affordable technology that can be distributed along with the traditional newspapers existing in newsstands.

With regard to improvements, the application still needs adjustments to not crash and the possibility of being used in 
any of the tablet or mobile manufacturers. Currently the app runs only on Android platforms. Criteria for usability and learning assessments will be made after application adjustments.

AR Musical will be tested with a wide range of students of different ages and the results of using the application will be presented in future work.

\section{REFERENCES}

[1] Cozza, R.; Durandm I.; Gupta, A. "Market Share: Ultramobiles by Region, OS and Form Factor, 4Q13 and 2013.” Gartner's website. Available at:

"http:/www.gartner.com/document/2672716". Last access: July 15th, 2014.

[2] Couse, L.J.; Chen, D.W. “A Tablet Computer for Young Children? Exploring its Viability for Early Childhood Education”. Journal of Research on Technology in Education, vol.10, no.1, 2010.

[3] M. Broadmeadow; Y. Mishida; G. Ledwich. "Digital tablets as a tool for blended learning in power engineering education”. In Proceedings of the 2013 Australasian Universities Power Engineering Conference (AUPEC), IEEE, University of Tasmania, Hobart, Australia, pp. 1-5.

[4] Travis, C.; Murano P. \&quot;A comparative study of the usability of touch-based and mouse-based interaction \& quot;. International Journal of Pervasive Computing and Communications, Vol. 10 no: 1, pp.115 - 134, 2014.

[5] Lane, N. D., Miluzzo, E., Lu, H., Peebles, D. Choudhury, T., \& Campbell, A. T. (2010). A survey of mobile phone sensing. IEEE Communications Magazine, 48(9), 140-150. doi:10.1109/MCOM.2010.5560598.

[6] Traxler, J. (2010). Will student devices deliver innovation, inclusion and transformation? Journal of the Research Centre for Educational Technologies, 6(1), 3-15.
[7] Piyare R.; Internet of Things: Ubiquitous Home Control and Monitoring System using Android based Smart Phone. International Journal of Internet of Things 2013; 2(1): 5-11. doi:10.5923.

[8] Martin G. F. Will massive open online courses change how we teach? Magazine Communications of the ACM, August 2012, 26-28. doi:10.1145/2240236.2240246.

[9] Reig, D. "MOOCs: A New Tool for the knowledges society". At the Center of Contemporary Culture of Barcelona's website. Available at:

"http://lab.cccb.org/en/moocs-a-new-tool-for-the-knowledgesociety/”. Last Access: February, 21st, 2017.

[10] T. P. Caudell; D. W. Mizell. Augmented Reality: An Application of Heads-Up Display Technology to Manual Manufacturing Processes. Proceedings of 1992 IEEE Hawaii International Conference on Systems Sciences, 1992, pp 659-669.

[11] Azuma, R. T., Baillot, Y., Behringer, R., Feiner, S., Julier, S., \& MacIntyre, B. (2001). Recent advances in augmented reality. IEEE Computer Graphics and Applications, 21(6), 34-47. doi:10.1109/38.963459.

[12] Milgram P.; Takemura H.; Utsumi A.; Kishino F. Augmented Reality: a class of displays on the reality-virtuality continuum. Proc. SPIE 2351, Telemanipulator and Telepresence Technologies, 282 (December 21, 1995); doi:10.1117/12.197321.

[13] FitzGerald E.; Ferguson R.; Adams A.; Gaved M.; Mor Y. and Thomas R. (2013). Augmented reality and mobile learning: the state of the art. International Journal of Mobile and Blended Learning, 5(4) pp. 43-58.

[14] Billinghurst, M. N., \& Henrysson, A. (2006). Research directions in handheld AR. International Journal of Virtual Reality, 2(1), 51-58.

[15] Brown K.; Prelude - An Augmented Reality iOS Application for Music Education (2014). Honors Projects. Paper 112.

[16] Ekuni, R.; Vaz, L.J.; Bueno, O.F. Levels of processing: the evolution of a framework, Psychol. Neurosci. 4(3), 2011 